\title{
EZH2 and Sonic Hedgehog Inhibition Reduce Proliferation, Migration, In Vitro Tumorigenesis, and CD133 Expression in Desmoplastic Medulloblastoma Cells
}

\author{
Javier de la Rosa ${ }^{1}$, Leire Tapia ${ }^{1}$, Mehdi H Shahi ${ }^{2}$, Bárbara Meléndez ${ }^{3}$, Juan A Rey ${ }^{4}$, Miguel A Idoate ${ }^{5}$ and Javier S Castresana ${ }^{*}$ \\ ${ }^{1}$ Department of Biochemistry and Genetics, University of Navarra School of Sciences, Pamplona, Spain \\ ${ }^{2}$ Faculty of Medicine, Interdisciplinary Brain Research Centre, Aligarh Muslim University, Aligarh, India \\ ${ }^{3}$ Molecular Pathology Research Unit, Virgen de la Salud Hospital, Toledo, Spain \\ ${ }^{4}$ IdiPaz Research Unit, La Paz University Hospital, Madrid, Spain \\ ${ }^{5}$ Department of Pathology, University of Navarra Clinic, Pamplona, Spain \\ *Corresponding author: Javier S Castresana, Department of Biochemistry and Genetics, University of Navarra School of Sciences, Pamplona, Spain; Tel: \\ +34948425600; E-mail: jscastresana@unav.es
}

Received date: June 01, 2017; Accepted date: June 09, 2017; Published date: June 16, 2017

Copyright: (c) 2017 Castresana JS, et al. This is an open-access article distributed under the terms of the Creative Commons Attribution License, which permits unrestricted use, distribution, and reproduction in any medium, provided the original author and source are credited.

\begin{abstract}
Medulloblastoma is the malignant brain tumor that most affects children and young people. Its treatment is very aggressive and can leave important neurocognitive sequelae in patients. Medulloblastoma can be classified histologically and molecularly in different subtypes. Our work focuses on the specific subtype in which the sonic hedgehog pathway is altered. DAOY cells, which correspond to desmoplastic Shh medulloblastoma, were independently treated with two pharmacological inhibitors: cyclopamine and DZNep. Cyclopamine directly inhibits Smo, thus inhibiting the sonic hedgehog pathway; while DZNep acts at the epigenetic level by inhibiting EZH2 function, a histone-lysine N-methyltransferase. The two inhibitions were compared cellularly and molecularly, demonstrating that both drugs reduced cell viability, colony formation, cell migration and the expression of cancer stem cells related genes, like CD133. In addition, the expression of different genes of the sonic hedgehog, EZH2, and other genes regulated by $\mathrm{EZH} 2$ and $\mathrm{GLI} 1$ were evaluated. The initial hypothesis, according to which the expression of EZH2 would regulate the sonic hedgehog pathway was not demonstrated. Quite the opposite, we observed that the sonic hedgehog pathway could positively regulate EZH2 expression. Our results, however, should be subjected to further experiments in order to elucidate the relationship between the epigenetic regulation exerted by $\mathrm{EZH} 2$ and the regulation of the sonic hedgehog pathway in medulloblastoma.
\end{abstract}

Keywords: Medulloblastoma; Sonic hedgehog; Cyclopamine; EZH2; DZNep

\section{Introduction}

Brain tumors are the most frequent solid tumors in childhood and the main cause of cancer death at that age. Medullobastoma is one of the most frequent pediatric tumors [1] that can appear not only in infancy but also in adults, although it presents peaks of greater incidence at 3 to 4 years and 5 to 9 years of age, being a rare disease in patients older than 50. There are genetic syndromes that can predispose to the development of medulloblastoma, like Gorling, Turcot, and Li-Fraumeni syndromes, although medulloblastomas associated to these syndromes happen in less than $7 \%$ of the cases [2]. The treatment of this embryonal tumor includes surgery, radiotherapy and chemotherapy, but due to the aggresiveness of these treatments patients suffer devastating neurocognitive sequelae [3]. For this reason, understanding the molecular mechanisms of the pathogenesis of medulloblastoma will help to find new specific treatments [1].

The World Health Organization (WHO) classifies medulloblastomas in the following histological subtypes: classical, desmoplastic/nodular, anaplastic, and large cell medulloblastomas $[4,5]$. According to the international consensus, the main signaling pathways related to the development of medulloblastoma are Wingless (Wnt) and sonic hedgehog (Shh), that constitute two of the four medulloblastoma subgroups. In addition, there are two other molecular subtypes: group
3 (usually presenting MYC amplification), and group 4 (linked to isochromosome $17 \mathrm{q}$ in more than $60 \%$ of cases, and mostly including those medulloblastoma not clearly belonging to other groups) [6].

\section{Shh meduloblastomas}

The subgroup of Shh medulloblastomas are produced by alterations in the sonic hedgehog signaling pathway. Alterations in this signaling pathway are thought to lead to the onset of several tumors [6]. The sonic hedgehog signaling pathway in vertebrates consists of the Patched receptor $(\mathrm{PTCH})$, a 12-transmembrane protein, and Smoothened (SMO) another 7-transmembrane protein-related receptor coupled to $G$ proteins. In mammals, there are three hedgehog gene families: Sonic (Shh), Indian (Ihh) and Desert (Dhh). Of these three, sonic hedgehog is the most studied in vertebrates [6].

In the absence of the Hh ligand, $\mathrm{PTCH}$ is localized on the cell membrane of the primary cilia and inhibits the function of SMO, which involves activation of GLI-1 and GLI-2 transcription, and inactivation of the GLI-3 transcriptional repressor (1). In the presence of the Hh ligand, PTCH is translocated and internalized, thereby eliminating its inhibitory effect on SMO which can now exert its function on the primary cilia membrane: activating the transcription of the GLI family of transcription factors by eliminating its binding to SUFU which has an inhibitory effect on the signaling pathway. GLI proteins are then activated and can enter the nucleus and thereby stimulate transcription of the target genes such as Gli1, Bcl-2, MYCN, 
Citation: Rosa JDL, Tapia L, Shahi MH, Meléndez B, Rey JA, et al. (2017) EZH2 and Sonic Hedgehog Inhibition Reduce Proliferation, Migration, In Vitro Tumorigenesis, and CD133 Expression in Desmoplastic Medulloblastoma Cells. J Brain Tumors Neurooncol 2: 113. doi: $10.4172 / 2475-3203.1000113$

Page 2 of 7

Bmil, Cyclin D2, PTCH1 and Hhip. Aberrant and altered signaling of the sonic hedgehog pathway leads to certain types of cancers and tumors [7].

Shh medulloblastomas correspond to approximately $25 \%$ of the cases, and mainly affect children between $0-3$ years and young adults older than 16 years $[1,6]$. The pathology of this molecular subtype is typically desmoplastic/nodular, although more than half of them may present classic features and rarely anaplastic features. Germline mutations of PTCH1 and SUFU have been associated with childhood medulloblastoma [6]. There are certain homeobox transcription factors such as Nkx2.2 and Pax6, that are related to the Shh pathway along neuronal development, and other molecules like Bmil which shows overexpression in human medulloblastoma [1]. SMO and SUFU genes are also occasionally mutated in human medulloblastoma [1,7]. A possible therapeutic strategy for the treatment of medulloblastoma might be the use of inhibitors of the sonic hedgehog pathway such as cyclopamine, a steroidal alkaloid isolated from a plant. Cyclopamine directly inhibits SMO, thus preventing Gli from binding to DNA and the consequent expression of its target genes [1].

\section{EZH2 (Enhancer of Zeste Homolog 2)}

EZH2 is the catalytic subunit of PRC2 (Polycomb Repressive Complex 2) that tri-methylates lysine 27 of histone H3. It is overexpressed in a wide variety of tumors and is related to advanced disease stage and bad prognosis [8,9]. High levels of EZH2 are associated with invasion and high proliferation rates in mammary carcinomas [8]. In addition, EZH2 has been shown to play an important role in the growth of prostate tumor stem cells [9]. EZH2 expression is also increased in adult and pediatric brain tumors [10], and confers poor prognosis. 3-Dezaneplanocin-A (DZNep) is capable of disrupting the activity of PRC2 by inhibiting the H3K27 trimethylation performed by EZH2 [11,12].
The objective of our work is to see how the hedgehog sonic pathway regulates the development and proliferation of medulloblastomas, and if inhibition of the pathway may conduct to reverting the cancer phenotype in medulloblastoma. We also want to study whether downregulation of EZH2 by its inhibitor DZNep modifies the sonic hedgehog pathway that is altered in Shh medulloblastoma. For this purpose, the DAOY medulloblastoma cell line was separately treated with cyclopamine and DZNep. The results obtained were compared cellularly and molecularly to see which of the two therapeutic options gave better results, if any. We also investigated whether EZH2 regulates the sonic hedgehog pathway since it is an epigenetic regulator that can modulate genes involved in different signaling pathways.

\section{Materials and Methods}

\section{Cell line}

DAOY, a desmoplastic medulloblastoma cell line, was used in our study. This cell line was obtained from the American Type Culture Collection, Manassas, VA, USA. For culture, RPMI L-Glutamax medium (Gibco-BRL, Gaithersburg MD, USA) was used with supplements as $10 \%$ fetal bovine serum (FBS), 1\% penicillin/ streptomycin, $0.1 \%$ amphotericin B (fungicide), and $4 \%$ non-essential amino acids. The cell line cultured in this medium was maintained in the incubator at $37^{\circ} \mathrm{C}$ with a $5 \% \mathrm{CO}_{2}$ atmosphere. Subcultures were performed after reaching $80-90 \%$ confluence by trypsinization. Another RPMI L-Glutamax medium supplemented with $2.5 \%$ fetal bovine serum (FBS) was used when the cells were treated with cyclopamine to prevent inactivation of the drug at high concentrations of FBS.

\begin{tabular}{|l|l|l|l|}
\hline Gen & Primer Forward $\left(\mathbf{5}^{\prime} \mathbf{- 3}^{\prime} \mathbf{)}\right.$ & Primer Reverse $\left(\mathbf{5}^{\prime} \mathbf{-} \mathbf{3}^{\prime} \mathbf{)}\right.$ & $\left.\mathbf{T}^{\mathbf{a}} \mathbf{(}^{\circ} \mathbf{C}\right)$ \\
\hline HPRT-RT & TGACACTGGCAAAACAATGCA & GGTCCTTTTCACCAGCAAGCT & 67 \\
\hline Gli1 q RT & CAAGTGCACGTTTGAAGGCT & CAACCTTCTTGCTCACACATGTAAG & 65.5 \\
\hline Gli3 a & ATGGACCCCAGGAATGGT & CAACCTTCTTGCTCACACATGTAAG & 64 \\
\hline SUFU & CCTCCAGATCGTTGGTGTCT & CCCCTCCGCATGTCAGTT & 65 \\
\hline Nkx2.2 & TCTACGACAGCAGCGACAAC & CGCCTTGGAGAAAAGCACTC & 65.1 \\
\hline PTH1 & CTTCGCTCTGGAGCAGATTT & CAGGACATTAGCACCTTCT & 55 \\
\hline EZH2 & CAGTAAAAATGTGTCCTGCAAGAA & TCAAGGGATTTCCATTTCTCTTTCGA & 66.9 \\
\hline CD133 & CAGAGTACAACGCCAAACCA & AAATCACGATGAGGGTCAGC & 64.6 \\
\hline TIMP3 & TCTGCAACTCCGACATCGT & TTGGTGAAGCCTCGGTACAT & 64.6 \\
\hline VASH1 & CATGGGAGGGCTTGATGAAGG & CAAGGTCAGCATGGACTAGGC & 67.8 \\
\hline
\end{tabular}

Table 1: Sequences and annealing temperatures of the oligonucleotides used for qRT-PCR.

\section{RNA extraction and retrotranscription}

RNA was extracted using the AllPrep DNA/RNA/Protein Mini Kit (Qiagen), following the protocol instructions. The concentration and purity of the RNA was determined by Nanodrop. The reverse transcription was performed by mixing in a DEPC-treated tube: $1 \mu \mathrm{g}$
RNA, $1 \mu$ l Random Primers (stock 250 ng/ $\mu \mathrm{l}$ ), $2 \mu \mathrm{l}$ dNTPs $(5 \mathrm{mM}$ stock) in a final volume of $12 \mu \mathrm{l} \mathrm{H20-DEPC.} \mathrm{Cells} \mathrm{were} \mathrm{incubated} \mathrm{for} 5$ min at $65^{\circ} \mathrm{C}$, and $4 \mu \mathrm{l}$ of $5 \mathrm{X}$ buffer and $2 \mu \mathrm{l}$ DTT were added. After 2 min incubation at $42^{\circ} \mathrm{C}, 1 \mu \mathrm{l}$ of SuperScript RT enzyme was added. The mixture was incubated for $10 \mathrm{~min}$ at $25^{\circ} \mathrm{C}, 50 \mathrm{~min}$ at $42^{\circ} \mathrm{C}$, and $15 \mathrm{~min}$ 
Citation: Rosa JDL, Tapia L, Shahi MH, Meléndez B, Rey JA, et al. (2017) EZH2 and Sonic Hedgehog Inhibition Reduce Proliferation, Migration, In Vitro Tumorigenesis, and CD133 Expression in Desmoplastic Medulloblastoma Cells. J Brain Tumors Neurooncol 2: 113. doi: $10.4172 / 2475-3203.1000113$

Page 3 of 7

at $70^{\circ} \mathrm{C}$. Finally, $99 \mu \mathrm{l}$ of sterile autoclaved water was added. The cDNA obtained was stored at $-20^{\circ} \mathrm{C}$ until use.

\section{qRT-PCR}

For PCR, the multicolor real-time PCR detection system IQ5 (BioRad, Hercules, CA, USA) was used. Each sample carried $12.5 \mu \mathrm{l}$ of SYBER green, $0.5 \mu \mathrm{l}$ of forward and reverse oligonucleotides (Table 1), and $10 \mu \mathrm{l}$ of $\mathrm{H}_{2} \mathrm{O}$. A masterbatch was prepared with SYBR green and $\mathrm{H}_{2} \mathrm{O}$ and mixed oligonucleotides. We made a sub-mix of each sample: $94 \mu \mathrm{l}$ of oligonucleotide mixture and $6 \mu \mathrm{l}$ of cDNA were added. Each well of the plate was charged with about $25 \mu \mathrm{l}$ by inverted pipetting to avoid bubbling. Triplicates of each sample were made. The program started with a first cycle of $95^{\circ} \mathrm{C}$ and $10 \mathrm{~min}$ for enzyme activity. The second cycle had three steps and about 40 repetitions ( $30 \mathrm{~s}$ at each step, and temperatures of 95,60 and $72^{\circ} \mathrm{C}$ ). To add the Melting Curve Analysis two cycles of one step each of 1 min were done at 95 and $72^{\circ} \mathrm{C}$ respectively. The melting curve consisted of a temperature rising every $30 \mathrm{~s}$ from about $0.5^{\circ} \mathrm{C}$ to about $90^{\circ} \mathrm{C}$. Each sample was measured in triplicate.

\section{MTT assay and cyclopamine and DZNep treatments}

After culture, DAOY cells were harvested and counted. The number of desired cells per well was about 5000 (final volume: $200 \mu$ l per well). Eight wells were seeded in a 96-well plate without the test product (in this case, DZnep and cyclopamine) as a control. Cells were incubated with more cyclopamine (dissolved in ethanol) medium in varying amounts: $0,1,2.5,5,10,15,30$, and $50 \mu \mathrm{M}$ for 24 and $48 \mathrm{~h}$. For DZNep the cells were cultured with medium plus DZnep (dissolved in DMSO) in variable amounts: $0,0.5,1,5,10$ and $15 \mu \mathrm{M}$ for 24,48 and $72 \mathrm{~h} .50 \mu \mathrm{l}$ of $2 \mathrm{mg} / \mathrm{ml} \quad$ MTT (3-(4,5-Dimethylthiazol-2-yl)-2,5diphenyltetrazolium bromide) was added to each well, including controls, and was incubated at $37^{\circ} \mathrm{C}$ for $2-3 \mathrm{~h}$. After that time, the black precipitate was made visible under the microscope, the medium was removed and $100 \mu \mathrm{l}$ of DMSO was added to each well including the controls. The plate was then allowed to stir gently for about 15-20 min at room temperature. Absorbance was read on a Multiskan plate reader at $540 \mathrm{~nm}$.

\section{Scratching assay for in vitro cell migration}

The DAOY cell monolayers of the treated and the untreated flasks were trypsinized with trypsin/EDTA (Gibco) and approximately 600,000 cells were cultured per well in a 6-well plate with $3 \mathrm{ml}$ of cell medium each. They were allowed to incubate at $37^{\circ} \mathrm{C}$ for about $24 \mathrm{~h}$ to reach high confluence (90\%). The next step consisted of making a line with a blue pipette tip on each well. The gap that was generated was inspected under the microscope over time to see and evaluate the movement of the cells and the filling of the wound produced. To correctly analyze cell migration, photographs were taken at $0,6,12,24$, 30 and $48 \mathrm{~h}$. The cells were maintained in the incubator at $37^{\circ} \mathrm{C}$ with a $5 \% \mathrm{CO} 2$ atmosphere. Cell migration was evaluated in the untreated (control) cells and in the cells treated with $15 \mu \mathrm{M}$ cyclopamine, and with 10 and $5 \mu \mathrm{M}$ DZNep.

\section{Colony formation assay}

The colony formation assay was performed to evaluate the ability of untreated and treated cells (with $15 \mu \mathrm{M}$ cyclopamine, 10 and $5 \mu \mathrm{M}$ DZNep) to create cell colonies in monolayer cultures. This experiment requires less time than the formation of colonies in soft agar. 1000
DAOY cells were cultured separately under the three conditions studied (control, cyclopamine and DZNep), in 6-well plates for 10 days along with $12 \mathrm{ml}$ of medium. After this time, the medium was discarded and the plates were washed with DPBS $(2 \mathrm{ml})$. Cells from each plate were fixed with $4 \mathrm{ml}$ of $4 \%$ paraformaldehyde for $30 \mathrm{~min}$, washed again with DPBS $(2 \mathrm{ml})$, and stained with $1 \%$ Crystal Violet (Sigma Aldrich, UK) for $15 \mathrm{~min}$. Finally, the plates were washed and allowed to dry for subsequent colony counts of each condition.

\section{In vitro tumorigenicity tested by the soft agar clonogenic assay}

By this assay the ability of the cells to form colonies without attachment can be assessed. This experiment was performed in DAOY control cells and in DAOY cells treated independently with $15 \mu \mathrm{M}$ cyclopamine, with $10 \mu \mathrm{M}$ and $5 \mu \mathrm{M}$ DZNep to evaluate their differences. Cells were mixed with $0.4 \%$ agar, and 5000 cells per well were plated onto 6-well plates that had solidified bottom layers of $1 \%$ agar. When the top layer of agar containing the cells was solidified, 2 $\mathrm{ml}$ of growth medium were added onto it. After 20 days, colonies were formed, and the medium was discarded. It was not necessary to fix the colonies because they were into the agar. They were stained with $250 \mu$ of $2.5 \%$ crystal violet (Sigma Aldrich, UK) for $5 \mathrm{~min}$, washed 4-5 times with DPBS, and counted.

\section{Statistical analysis}

Statistical analysis was performed using GraphPad Software Inc (California, USA). Student's t-test was chosen to compare the colonies obtained in both treatments (cyclopamine and DZNep) versus their respective controls. The same analysis was carried out after qRT-PCR. Data represents means $\pm \mathrm{SD}$ (standard deviation). The results are considered significant when the value of $\mathrm{p}$ is less than $0.05\left(^{*}\right)$, very significant when $\mathrm{p}$ is less than $0.01\left(^{* *}\right)$, highly significant when $\mathrm{p}$ is less than $\left.0.001{ }^{(* *}\right)$ and extremely significant when $p$ is less than $\left.0.0001{ }^{* * * *}\right)$.

\section{Results}

\section{Cyclopamine and DZNep reduce viability of DAOY medulloblastoma cells}

We performed an MTT colorimetric assay to know the IC50 of cyclopamine and DZNep. IC50 is the inhibitory concentration at which half of the cells die from the drug's effect. DAOY cells were treated with cyclopamine and DZNep at different concentrations: 0 to $50 \mu \mathrm{M}$. In the case of cyclopamine, the concentration of $15 \mu \mathrm{M}$ was chosen as its IC50 (Figure 1). With the DZNep even though this test was performed a few times, no good results were obtained. For this reason, it was decided to choose concentrations of 10 and $5 \mu \mathrm{M}$ as their IC50. Molecular and cellular experiments were performed on cyclopamine-treated DAOY cells after $48 \mathrm{~h}$ of exposure and DAOY cells treated with DZNep after $72 \mathrm{~h}$ of exposure according to results published elsewhere [20,21].

\section{In vitro tumorigenesis decays in DAOY medulloblastoma cells after inhibiting the sonic hedgehog pathway and EZH2}

Ethanol-treated control DAOY cells and cyclopamine-treated DAOY cells were cultured in 6-well Petri dishes. After 10 days we 
Citation: Rosa JDL, Tapia L, Shahi MH, Meléndez B, Rey JA, et al. (2017) EZH2 and Sonic Hedgehog Inhibition Reduce Proliferation, Migration, In Vitro Tumorigenesis, and CD133 Expression in Desmoplastic Medulloblastoma Cells. J Brain Tumors Neurooncol 2: 113. doi: $10.4172 / 2475-3203.1000113$

Page 4 of 7

observed that the number of colonies were reduced in the treated cells with respect to the control cells (Figure 2).

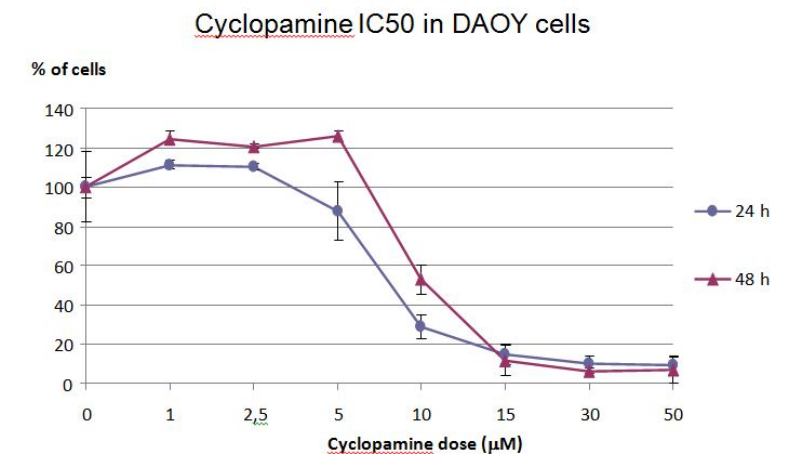

Figure 1: Cyclopamine reduces cell viability of DAOY medulloblastoma cells. The graph shows the effect of cyclopamine dissolved in ethanol on DAOY cells at different concentrations: 0,1 , $5,10,15,30$ and $50 \mu \mathrm{M}$. The effect of cyclopamine on DAOY cells is shown after 24 and $48 \mathrm{~h}$ of exposure. The concentration of $15 \mu \mathrm{M}$ is taken as the IC50 of cyclopamine.

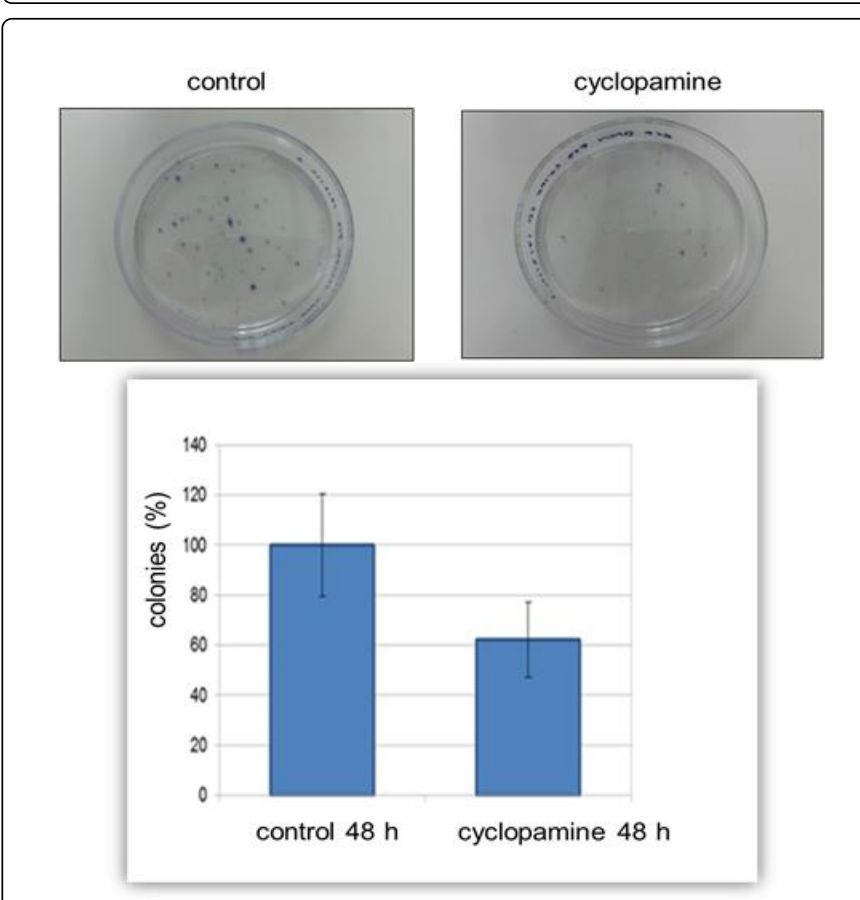

Figure 2: Images of a control plate and a plate with cyclopaminetreated DAOY cells. It is easily observable how cyclopamine reduces the ability of DAOY cells to adhere to the plate and form colonies. Graphs show the effect of cyclopamine on the ability to form colonies of DAOY cell lines. The colonies were counted visually in all cases.

DMSO-treated control DAOY cells and $10 \mu \mathrm{M}$ DZNep treated DAOY cells were also cultured. No colony was observed after 10 days in DAOY cells treated with $10 \mu \mathrm{M}$ DZNep (Figure 3). The plates were stained with $1 \%$ crystal violet to observe the colonies formed along 10 days. Colonies were counted visually in all cases (Figures 2 and 3). It can be concluded that inhibitory treatments with these drugs reduce the ability of Daoy medulloblastoma cells to adhere and form colonies.

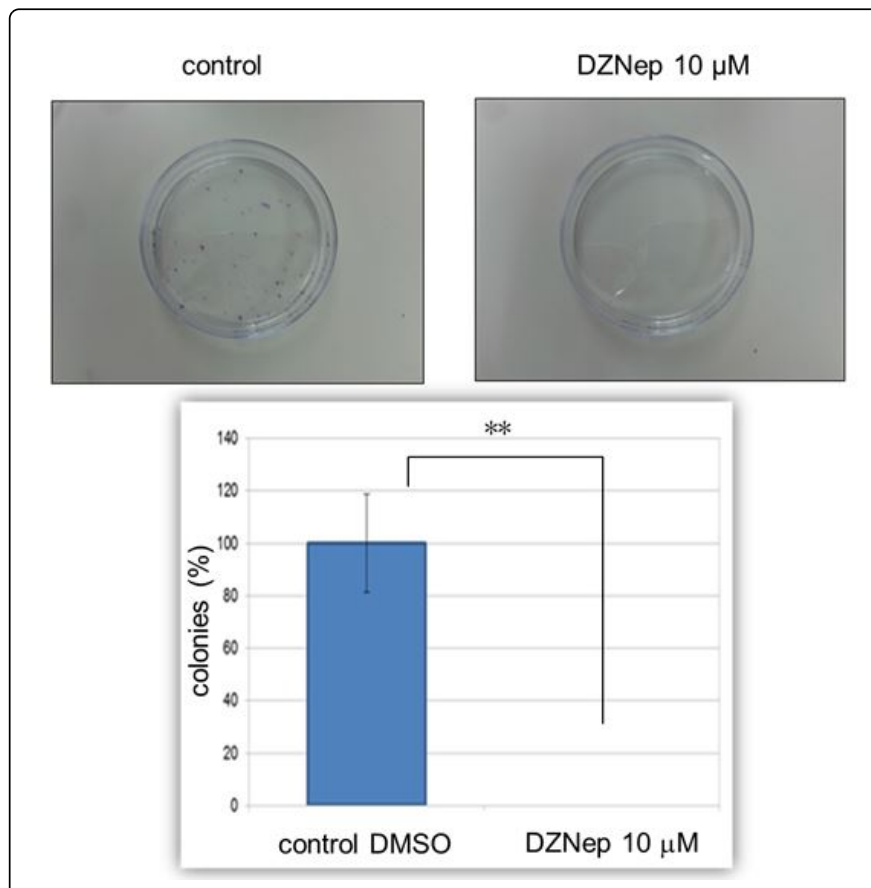

Figure 3: Images of a control plate and a plate with DAOY cells treated with $10 \mu \mathrm{M}$ of DZNep. The effect of DZNep is greater than the effect shown by cyclopamine, since there is no colony formed on DZNep-treated cells. Graphs show the effect of DZNep on the ability to form colonies of DAOY cell lines. The colonies were counted visually in all cases. Significant results: $p<0.01\left(^{(*)}\right.$.

In order to analyze tumorigenicity in vitro, the ability of these DAOY cells to form colonies in soft agar was also studied. In this way the ability of cells to form colonies without adherence to a surface was observed. The colonies formed (Figure 4) were counted visually with the help of a magnifying glass. In DAOY cells with ethanol and DMSO control, a greater number of colonies were observed compared to those treated with cyclopamine and DZNep. In the case of DAOY cells treated with $10 \mu \mathrm{M}$ DZNep for $72 \mathrm{~h}$ the number of colonies observed was lower than those treated with $15 \mu \mathrm{M}$ cyclopamine for $48 \mathrm{~h}$ (Figure $4)$.

\section{Cyclopamine and DZNep reduce migration of DAOY medulloblastoma cells}

To continue with the study of in vitro tumorigenicity in DAOY cells the scratching assay, a simple test used to measure cell migration, was carried out. DAOY cells (control cells with ethanol, $15 \mu \mathrm{M}$ cyclopamine cells, DMSO control cells and 10 and $5 \mu \mathrm{M}$ DZNep cells) were plated in 6-well plates and allowed to incubate for about $24 \mathrm{~h}$ to reach confluence. Then, a line was made on the surface of cells with a pipette tip. From that moment, cell migration was studied for about 48 h. The cells tended to move towards the wound, closing it in all the conditions of our work. However, there were differences between cells treated with cyclopamine and their respective control as well as between cells treated with DZNep and its control. In both cases, the inhibitory treatments reduced cell migration, and the initial confluence 
Citation: Rosa JDL, Tapia L, Shahi MH, Meléndez B, Rey JA, et al. (2017) EZH2 and Sonic Hedgehog Inhibition Reduce Proliferation, Migration, In Vitro Tumorigenesis, and CD133 Expression in Desmoplastic Medulloblastoma Cells. J Brain Tumors Neurooncol 2: 113. doi: $10.4172 / 2475-3203.1000113$

Page 5 of 7

was also diminished. In DAOY cells treated with DZNep at both 10 and $5 \mu \mathrm{M}$ the confluence decreased much more after $48 \mathrm{~h}$ than in cells treated with cyclopamine (Figure 5).
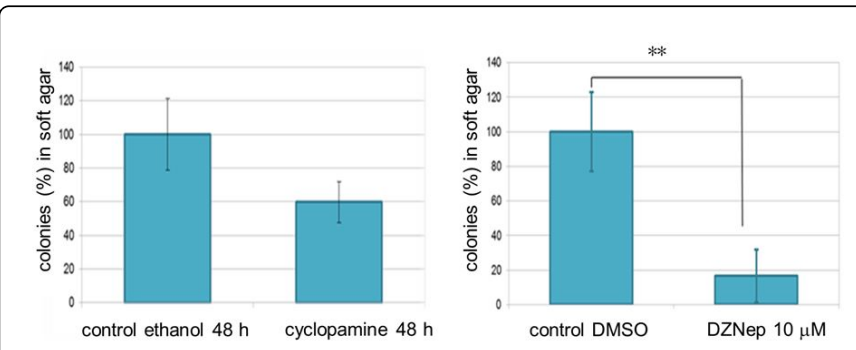

Figure 4: Effect of cyclopamine and DZNep on the ability to form colonies on soft agar. Percentage of colonies formed after treatment with cyclopamine at $15 \mu \mathrm{M}$ for $48 \mathrm{~h}$ versus corresponding control with ethanol, and after treatment with DZNep at $10 \mu \mathrm{M}$ for $72 \mathrm{~h}$ against their corresponding control with DMSO. Significant results: $\mathrm{p}<0.01$ in the treatment with DZNep.

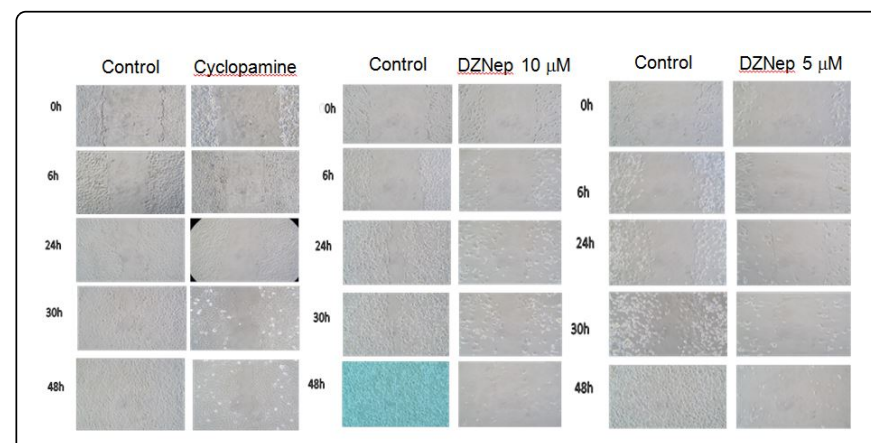

Figure 5: Scratching assay to determine cell migration in vitro. Cyclopamine at $15 \mu \mathrm{M}$ decreased cell migration. The photos were taken at $0,6,24,30$ and 48 h. Cyclopamine study: cells used correspond to control cells with ethanol and to cells treated with 15 $\mu \mathrm{M}$ cyclopamine for $48 \mathrm{~h}$. DZNep study: cells used were control cells with DMSO, and cells with DZNep at $10 \mu \mathrm{M}$ for $72 \mathrm{~h}$ or cells with DZNep at $5 \mu \mathrm{M}$ for $48 \mathrm{~h}$.

\section{qRT-PCR expression levels of sonic hedgehog pathway regulators and related genes}

After treatment with cyclopamine and DZNep, we determined the expression of Glil and some of the Gli1 target genes (Figures 6-8), such as Nkx2.2 and PTCH1 (Figure 7). In addition, other important components of the sonic hedgehog pathway, such as SUFU and Gli3a (Figure 6), were studied. Expression of EZH2 (Figure 8) was also determined by qRT-PCR since DZNep inhibits EZH2. CD133 expression was also studied (Figure 9) as it is considered to be a cancer stem cell maker. Finally, we also included the expression of EZH2 target genes such as TIMP3 and VASH1 (Figure 8) both negative regulators of angiogenesis. Cyclopamine decreased Glil expression which also led to decreased expression of $\mathrm{Nkx} 2.2, \mathrm{PTCH} 1$ and in turn SUFU and Gli3a. Expression of CD133, EZH2 and its target genes TIMP3 and VASH1 was also reduced after treating the cells with cyclopamine.

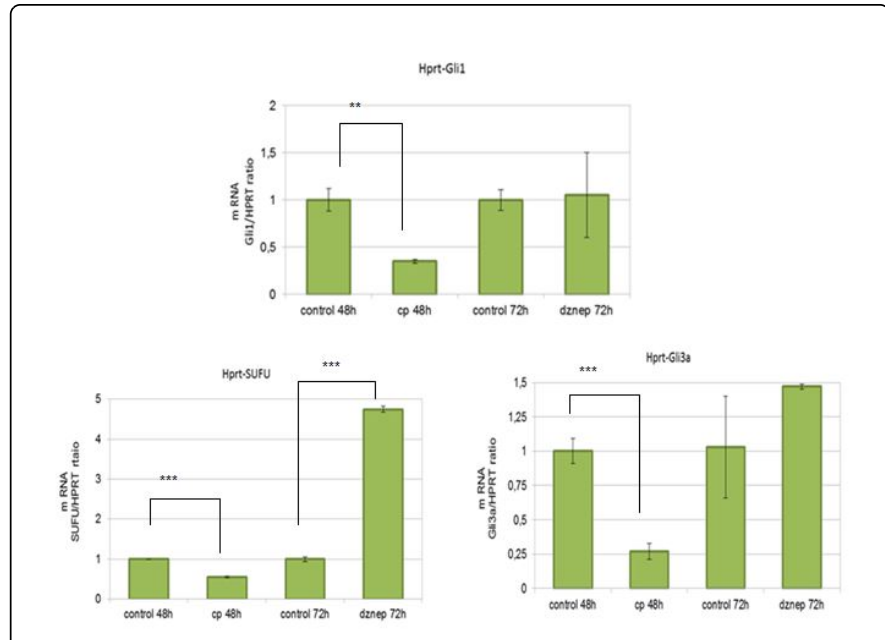

Figure 6: Expression of Gli1, Gli3a, and SUFU genes after treating the DAOY cell line with cyclopamine and DZNep. The graphs show the change in expression that was observed between the genes studied versus the expression of the HPRT gene that was taken as a reference. Significant result $\mathrm{p}<0.05\left({ }^{*}\right)$, very significant $\mathrm{p}<0.01\left({ }^{* *}\right)$ highly significant $\left.\mathrm{p}<0.001{ }^{* * *}\right)$ and extremely significant $\mathrm{p}<0.0001$ $\left({ }^{* * *}\right)$.

Quite the contrary, DZNep treatment neither decreased Gli1, Nkx2.2, PTCH1, SUFU and Gli3a expression (Figures 6 and 7) nor decreased EZH2 expression or the expression of its target genes TIMP3 and VASH1 (Figure 8). However, both DZNep and cyclopamine reduced CD133 expression (Figure 9).

\section{Discussion}

Meduloblastoma is a brain tumor of very poor prognosis whose treatment consists basically in surgery, radiotherapy and chemotherapy which can leave important sequelae in children [3]. For this reason research on molecular therapies is important to find alternative or complementary ways to make the treatment of medulloblastomas more effective. With this idea in mind we decided to treat the DAOY medulloblastoma cell line with two drugs acting at different levels: cyclopamine and DZNep. Cyclopamine inhibits the sonic hedgehog pathway by directly inhibiting SMO, while DZNep acts at the epigenetic level as an inhibitor of EZH2, a histone 3 lysine $27 \mathrm{~N}$ methyltransferase.

By an MTT assay, cyclopamine was found to decrease the viability of the DAOY cells. By this assay $15 \mu \mathrm{M}$ was taken as the IC50 of cyclopamine in these cells (Figure 1), in agreement with other works [1]. For DZNep the IC50 and exposures to the drug were obtained from the literature $[13,14]$. The use of both drugs independently on the DAOY line reduced the ability of these cells to divide and form colonies under adherent and non-adherent conditions. Cells treated with DZNep could not form colonies at all on adherent surfaces (Figure 3). However, some colonies were observed in the colony formation assay on soft agar (Figure 4). The number of colonies formed after treatment with DZNep was lower compared to cyclopamine-treated DAOY cells (Figure 4). Similar results have been presented elsewhere in EZH2 inhibitory experiments [15]. 
Citation: Rosa JDL, Tapia L, Shahi MH, Meléndez B, Rey JA, et al. (2017) EZH2 and Sonic Hedgehog Inhibition Reduce Proliferation, Migration, In Vitro Tumorigenesis, and CD133 Expression in Desmoplastic Medulloblastoma Cells. J Brain Tumors Neurooncol 2: 113. doi: $10.4172 / 2475-3203.1000113$

Page 6 of 7

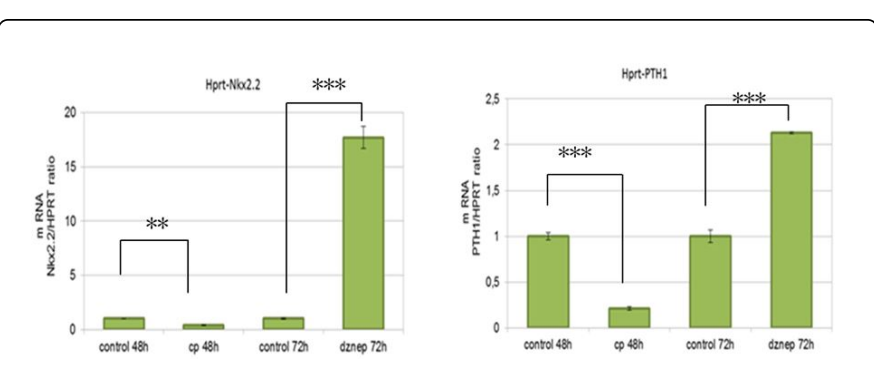

Figure 7: Expression of Nkx2.2 and PTCH1 genes after treating the DAOY cell line with cyclopamine and DZNep. The graphs show the change in expression that was observed between the genes studied versus the expression of the HPRT gene that was taken as a reference. Significant result $\mathrm{p}<0.05\left({ }^{*}\right)$, very significant $\mathrm{p}<0.01\left({ }^{* *}\right)$ highly significant $\mathrm{p}<0.001\left({ }^{* *}\right)$ and extremely significant $\mathrm{p}<0.0001$ $\left({ }^{* * *}\right)$.

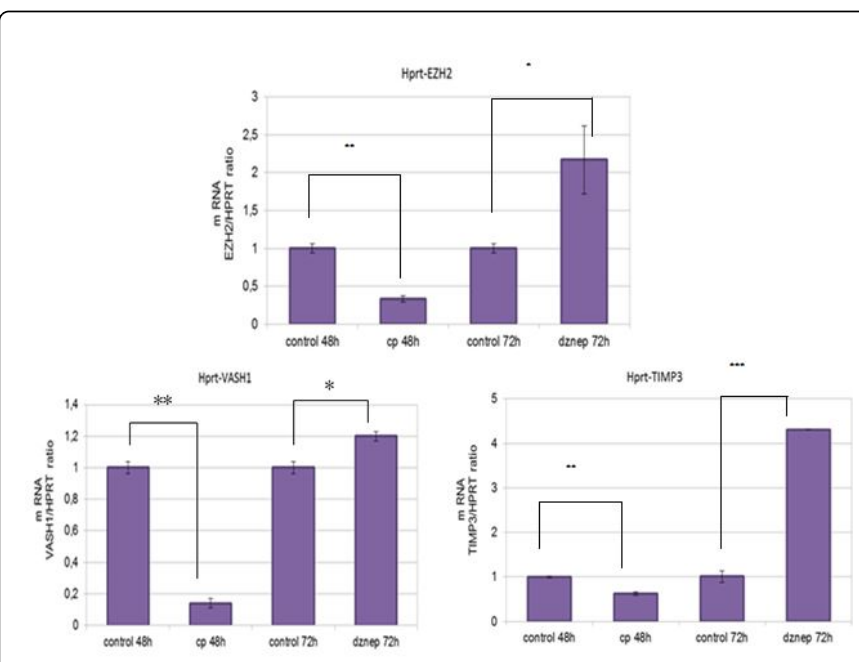

Figure 8: Expression of EZH2, VASH1 and TIMP3 genes after treating the DAOY cell line with cyclopamine and DZNep. The graphs show the change in expression that was observed between the genes studied versus the expression of the HPRT gene that was taken as a reference. Significant result $\mathrm{p}<0.05\left(^{*}\right)$, very significant $\mathrm{p}<0.01 \quad(* *)$ highly significant $\mathrm{p}<0.001 \quad(* * *)$ and extremely significant $\left.\mathrm{p}<0.0001{ }^{* * * *}\right)$.

Medulloblastoma is an invasive tumor that can spread through the cerebrospinal fluid and metastasize to different areas of the brain and spinal cord [16]. We observed that DAOY cells treated with cyclopamine and DZNep reduced cell migration compared to their respective controls (Figure 5). Furthermore, in the case of DZNep, not only cell migration but also cell confluence was reduced. The results obtained in both treatments were satisfactory since both treatments managed to reduce cell growth, the formation of colonies and cellular migration.

In order to complete the objective of this work, the expression of several Shh and related genes was analyzed by qRT-PCR. Although our initial theory was that EZH2 could somehow regulate the sonic hedgehog pathway, the results obtained proved that this is not so, but the other way around. Cyclopamine, an SMO antagonist, significantly reduced the expression of Gli1, Nkx2.2, PTCH1, Gli3a and SUFU (Figures 6 and 7). Our results are then similar to results published elsewhere on Shh pathway inhibition by cyclopamine [1]. However, in DAOY cell lines inhibited with DZNep, an EZH2 inhibitor, neither Gli1 nor its target genes reduced their levels of expression (Figure 8). Even, surprisingly, DZNep did not decrease EZH2 mRNA levels (Figure 8), which makes us think that perhaps DZNep acts at the level of the protein preventing it from performing its function, rather than at the transcription level of the EZH2 gene. On the contrary, EZH2 did reduce its expression after treatment with cyclopamine (Figure 8), reason that leads us to think that the sonic hedgehog pathway might be regulated by EZH2 in some way still unknown.

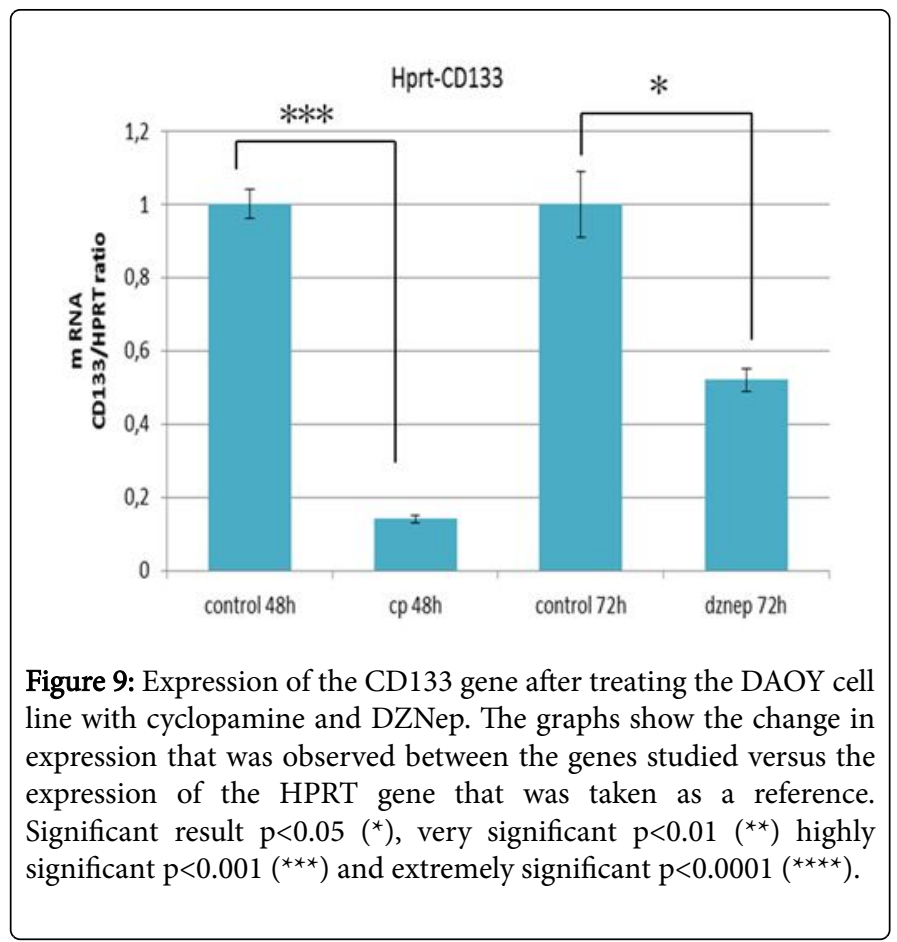

Another gene that was studied was CD133. Many works show that CD133 is a surface marker of tumor stem cells. CD133 has been found in many types of cancers such as brain, lung, colon, liver, kidney. According to several works, the CD133+ cells constitute the subpopulation with tumor stem cells characteristics such as differentiation capacity and self-renewal [17]. Cyclopamine and DZNep significantly reduced CD133 expression meaning that tumor stem cells were affected by these drugs (Figure 9). This is an important fact since tumor stem cells are usually resistant to conventional therapies and are responsible for tumor recurrence [18].

Some target genes of EZH2, such as VASH1 and TIMP3 [19], were also studied. In cells treated with cyclopamine their levels were significantly reduced whereas in cells treated with DZNep there was no decrease in their expression (Figure 8). VASH1 is an inhibitor of tumor growth and angiogenesis that inhibits the migration, proliferation and formation of endothelial cell networks [20]. Their expression was not reduced in cells treated with DZNep compared to those treated with cyclopamine, which would explain that the treatment with DZNep showed lower migration and confluence than the cyclopamine treatment (Figure 8), probably due to the fact that DZNep did not inhibit VASH1 and TIMP3 expression, which then could act as negative regulators of migration and metastatic capacity. TIMP3 is an 
Citation: Rosa JDL, Tapia L, Shahi MH, Meléndez B, Rey JA, et al. (2017) EZH2 and Sonic Hedgehog Inhibition Reduce Proliferation, Migration, In Vitro Tumorigenesis, and CD133 Expression in Desmoplastic Medulloblastoma Cells. J Brain Tumors Neurooncol 2: 113. doi: $10.4172 / 2475-3203.1000113$

Page 7 of 7

inhibitor of metalloproteinases, enzymes which are responsible for degrading the extracellular matrix and inducing migration and metastases. TIMP3 is also an inhibitor of angiogenesis, a phenomenon that leads to neovascularization and tumor progression [21]. TIMP3 expression was reduced in cyclopamine-treated DAOY cells but was maintained higher in DZNep-treated DAOY cells (Figure 8). That TIMP3 had its reduced expression in DAOY cells treated with DZNep corroborated that these cells had lower migration capacity than those treated with cyclopamine.

\section{Conclusions}

We may highlight the following points as conclusions of our work:

Cyclopamine and DZNep separately decreased in vitro tumorigenesis in DAOY medulloblastoma cells by reducing cell viability, cell migration, and colony formation in plaques and in soft agar. Therefore, not only the sonic hedgehog pathway but also EZH2 plays an important role in Shh medulloblastoma cell growth regulation. This suggests that EZH2 might be a potential therapeutic target against Shh medulloblastoma.

Contrary to what was initially expected, EZH2 does not seem to regulate the sonic hedgehog pathway, but rather the opposite. When the sonic hedgehog pathway is inhibited by cyclopamine, so does the expression of EZH2, although the expression of its target genes VASH1 and TIMP3 is not increased as might be expected. However, it appears that DZNep does not affect EZH2, TIMP3 and VASH1 expression, which indicates that DZNep might inhibit EZH2 enzymatic function but not EZH2 mRNA levels. Treatment with DZNep does not reduce the expression of genes of the sonic hedgehog pathway or other genes related to it. Our results suggest there is some kind of relationship between the sonic hedgehog pathway and EZH2, possibly consisting on a positive regulation executed by sonic hedgehog on EZH2 expression, although it is necessary to investigate it in depth for proper understanding, since it seems to be a complex relationship.

Finally, DZNep and cyclopamine reduced CD133 expression in DAOY cells, which means that both treatments somehow reduce the subpopulation of cancer stem cells in Shh medulloblastoma.

\section{Acknowledgements}

This research was supported in part by grants from Fundación Universitaria de Navarra, Pamplona, and Fondo de Investigación Sanitaria, Madrid: PI13/00055 (JAR), and PI13/00800 (BM).

\section{References}

1. García-López R, Vera-Cano B, Vacas-Oleas A, de la Rosa J, Gallo-Oller G, et al. (2013) Sonic hedgehog inhibition reduces in vitro tumorigenesis and alters expression of Gli1-target genes in a desmoplastic medulloblastoma cell line. J Cancer Res Ther 1: 11-23.

2. Taylor MD, Mainprize TG, Rutka JT (2000) Molecular insight into medulloblastoma and central nervous system primitive neuroectodermal tumor biology from hereditary syndromes: a review. Neurosurgery 47 : 888-901.
3. Rutkowski S, von Hoff K, Emser A, Zwiener I, Pietsch T, et al. (2010) Survival and prognostic factors of early childhood medulloblastoma: an international meta-analysis. J Clin Oncol 28: 4961-4968.

4. Louis DN, Ohgaki H, Wiestler OD, Cavenee WK, Burger PC, et al. (2007) The 2007 WHO classification of tumours of the central nervous system. Acta Neuropathol 114: 97-109.

5. Louis DN, Perry A, Reifenberger G, von Deimling A, Figarella-Branger D, et al. (2016) The 2016 World Health Organization Classification of Tumors of the Central Nervous System: a summary. Acta Neuropathol 131: 803-820.

6. Taylor MD, Northcott PA, Korshunov A, Remke M, Cho YJ, et al. (2012) Molecular subgroups of medulloblastoma: the current consensus. Acta Neuropathol 123: 465-472.

7. Abidi A (2014) Hedgehog signaling pathway: a novel target for cancer therapy: vismodegib, a promising therapeutic option in treatment of basal cell carcinomas. Indian J Pharmacol 46: 3-12.

8. Collett K, Eide GE, Arnes J, Stefansson IM, Eide J, et al. (2006) Expression of enhancer of zeste homologue 2 is significantly associated with increased tumor cell proliferation and is a marker of aggressive breast cancer. Clin Cancer Res 12: 1168-1174.

9. Varambally S, Dhanasekaran SM, Zhou M, Barrette TR, Kumar-Sinha C, et al. (2002) The polycomb group protein EZH2 is involved in progression of prostate cancer. Nature 419: 624-629.

10. Crea F, Hurt EM, Farrar WL (2010) Clinical significance of Polycomb gene expression in brain tumors. Mol Cancer 9: 265.

11. Crea F, Hurt EM, Mathews LA, Cabarcas SM, Sun L, et al. (2011) Pharmacologic disruption of Polycomb Repressive Complex 2 inhibits tumorigenicity and tumor progression in prostate cancer. Mol Cancer 10: 40.

12. Tan J, Yang X, Zhuang L, Jiang X, Chen W, et al. (2007) Pharmacologic disruption of Polycomb-repressive complex 2-mediated gene repression selectively induces apoptosis in cancer cells. Genes Dev 21: 1050-1063.

13. Choudhury SR, Balasubramanian S, Chew YC, Han B, Marquez VE, et al. (2011) (-)-Epigallocatechin-3-gallate and DZNep reduce polycomb protein level via a proteasome-dependent mechanism in skin cancer cells. Carcinogenesis 32: 1525-1532.

14. Ciarapica R, Carcarino E, Adesso L, De Salvo M, Bracaglia G, et al. (2014) Pharmacological inhibition of EZH2 as a promising differentiation therapy in embryonal RMS. BMC Cancer 14: 139.

15. Alimova I, Venkataraman S, Harris P, Marquez VE, Northcott PA, et al. (2012) Targeting the enhancer of zeste homologue 2 in medulloblastoma. Int J Cancer 131: 1800-1809.

16. Schroeder K, Gururangan S (2014) Molecular variants and mutations in medulloblastoma. Pharmgenomics Pers Med 7: 43-51.

17. Ren F, Sheng WQ, Du X (2013) CD133: a cancer stem cells marker, is used in colorectal cancers. World J Gastroenterol 19: 2603-2611.

18. Beier D, Schulz JB, Beier CP (2011) Chemoresistance of glioblastoma cancer stem cells--much more complex than expected. Mol Cancer 10: 128.

19. Crea F, Fornaro L, Bocci G, Sun L, Farrar WL, et al. (2012) EZH2 inhibition: targeting the crossroad of tumor invasion and angiogenesis. Cancer Metastasis Rev 31: 753-761.

20. Yoshinaga K, Ito K, Moriya T, Nagase S, Takano T, et al. (2008) Expression of vasohibin as a novel endothelium-derived angiogenesis inhibitor in endometrial cancer. Cancer Sci 99: 914-919.

21. Baker AH, Edwards DR, Murphy G (2002) Metalloproteinase inhibitors: biological actions and therapeutic opportunities. J Cell Sci 115: 3719-3727. 